\title{
Digital Image Watermarking based on LSB Techniques: A Comparative Study
}

\author{
Munirah Gaaed Almutiri \\ Department of Computer Science \\ College of Computer \\ Qassim University
}

\author{
Mohamed Tahar Ben Othman \\ Department of Computer Science \\ College of Computer \\ Qassim University
}

\begin{abstract}
Information security is one among the top level issues that have being addressed since a decade and intensively focused on nowadays. As owning the information is considered having the power these days, maintaining this information secure is among the only ways to maintain this power. During the last decade, several techniques for information security were under research. One of these areas is the security of information communication. Among the techniques handling this issue is the digital image watermarking. The aim of this paper is to present several works in this area and compare the performance of such techniques. In this paper we focus on Least Significant Bit (LSB) techniques in spatial domain, starting with a description of the latest work carried out over LSB and ending with making a comparison between various LSB watermarking schemes.
\end{abstract}

\section{General Terms}

Digital Image watermarking

\section{Keywords}

Watermarking, LSB, least significant bit, spatial domain, gray images, information security, digital image.

\section{INTRODUCTION}

Because the Internet makes a huge availability of multimedia over the world, the information becomes easier for duplication and distribution. That is why protection has become very important. Thus, for hiding multimedia information, watermarking is used and can be considered as relatively new technique. Digital Watermarking can be used in different applications, including Copyright protection, Authentication, broadcast monitoring, etc. Digital watermarking can be defined as a technique used for hiding the information in multimedia data to secure the original data from attacks and protect the ownership right. Digital image watermarking is an effective method for embedding the watermarks in the image to enhance the security of the digital content and to protect the data from unauthorized use and the ownership right for the digital data. The watermarking techniques are trying to embed a binary logo images (hidden information) which should be difficult to remove and/or detect to the image processing techniques like cropping, blurring, geometrical transformations, or even lossy compression techniques.

The image watermarking system is divided into two processes: embedding of a watermark into the cover image and extraction of the watermark from the image. Embedding procedure is done at the source end, by using any embedding watermarking algorithm to insert the watermark into the original (called also cover or host) image resulting on a watermarked image. In the extraction process, a watermarking extraction algorithm is used to extract the watermark from the watermarking image.
There are many algorithms, which are used for embedding process. These algorithms can be categorized according to working domain into: Spatial domain and Frequency domain. Spatial domain techniques embed the watermarks by simply changing the values of some selected pixels of the over image. Examples of spatial domain techniques are Least Significant Bit (LSB) Modification, Patchwork and fractal compression. In Frequency domain, the watermark information is embedded in the transform domain. Frequency watermarking algorithm converts the original image using a predefined transformation. Then the watermark data is embedded in the transformed image. The implementation of watermark is difficult and computationally expensive. The most common transformation techniques used in frequency domain are Fourier Transform (DFT), Discrete Cosine Transform (DCT) and Discrete Wavelet Transform (DWT). Each of these techniques has its own properties and represents the image in different ways.

In this paper we will focus on Least Significant Bit (LSB), which is a spatial domain technique. The embedding of the watermark into the original image is done by selecting a subset of pixels and substituting the least significant bit of the selected pixels with the watermark bits. The LSB techniques, are easy to implement and requires a little computation cost for both embedding and extraction processes. On the hand, they are sensitive to signal processing operations and generally show reduced robustness to different attacks. Even if there is a wide number of proposed LSB algorithms, still this area needs a lot of research, as still there is a lack of solid solution. The aim of this paper is to provide a set of the different proposed LSB algorithms, compare them and point out the common strengths and main gaps that should be focused on.

The remaining of this paper is divided as follows: in Section 2 , we present the related work which focus on a review of LSB digital image watermarking. Section 3 defines the LSB selected algorithms. Section 4 describes the differences between these algorithms provided by their authors and Section 5 presents our measurements, results and discussion.

\section{RELATED WORKS}

Several researchers were conducted for digital image watermarking. Some of those using LSB techniques are studied and briefly described in this section. For invisible digital watermarking there are many algorithms available. The simplest algorithm is Least Significant Bit (LSB), in which the bits of the watermark image replace the least significant bits (LSB) of the cover image.

Yang et al. [3] designed a novel testing and verifying method of digital images using the PKI (Public-Key Infrastructure), PublicKey Cryptography and watermark techniques. The main idea of the paper is to encrypt the watermark image before embed it into the least significant bits of cover images to preserve the 
authenticity of the evidence. Hong Jie He et al. proposed in [4] the use of the discrete wavelet transform (DWT) to generate watermark image before embedding into the cover image by using LSB. This proposed method enhances the protection of the watermarking system. Sung-Cheal Byun et al. in [5] proposed a fragile watermarking algorithm for the authentication of image. They use singular value decomposition (SVD) of the image to see the integrity of images. With a specific end goal to make authentication data, the singular value is converted into binary bits by modular arithmetic before embedding it into the least significant bits of the cover image. Gil-Je Lee et al. proposed another LSB watermarking schema in [6] by using random mapping function. The idea of proposed algorithm is to make random coordinate of cover image by using random mapping function then embedding watermark using the LSB. The proposed schema increases the robustness of the watermarked image. Bamatraf A. et al. in [8] proposed a new LSB scheme with embedding watermark image in third and fourth LSB so it will be more secure against the attacks impacting the 2 least significant bits. Singh K. K. et al. in [9] uses a combined approach based on both digital watermarking and cryptography for embedding the secret information in order to achieve higher security and efficiency. The proposed method used an asymmetric key cryptography technique called RSA and a spatial domain technique in digital watermarking. El Kerek B. et al. described in [11] a new method that combines between the traditional LSB and the hamming code algorithm to increase the robustness of the watermark against attacks. The results based on block matching algorithm, the LSB and Hamming code showed a high compression rate of $98 \%$, and a high immunity against noise. In [12] Bamatraf, proposed a new digital watermarking algorithm using the least significant bit (LSB) by inversing the watermark bits before embedding into the cover image. The proposed algorithm increases the security of watermarking. No one will expect the watermark bit was inversed. Kumar S. et al. introduces in [13] the concept of a modified LSB using SVD. The modified LSB uses the image compression algorithm. The watermark image is compressed before be embedded in the cover image using Singular Value Decomposition (SVD) technique, which aims to compress the watermark image. Comparing the proposed method with the traditional LSB, the first gives better results. Mohamed T.B.O. in [16-19] provided an LSB watermarking technique that takes into account some attacks mainly rotation and cropping attacks. The proposed technique called Content Addressing Method (CAM) is used to embed each set of bits of the watermark into several pixels (Cluster). These pixels have the same content address in the cover image. By using this duplication, the impact of the attack is reduced a lot.

\section{SELECTED LSB ALGORITHMS}

The aim of this paper is to implement some of watermarking algorithms based on LSB technique that the researches have done starting with the traditional LSB algorithm, where the most significant bit (MSB) of the watermark image is embedded into the least significant bit (LSB) of the cover image to act as basic comparison for the other algorithms.

\subsection{Algorithm 1}

The idea behind traditional LSB watermarking technique [1] is the following: select the cover and watermark images. The latter is a gray-scale image, which is transferred to binary values. Then change the value of the LSB of the cover image in every pixel with the MSB of watermark image, which results into a watermarked image. The extraction process is the procedure of the watermark retrieval.

\section{Embedding Algorithm}

1. Read the cover image

2. Read the watermark image to hide in the cover image

3. Determine the size of cover image and the size of watermark

4. Set the LSB of cover image to the value of the MSB of watermark

5. Generate watermarked image

\section{Extraction Algorithm}

1. Read in watermarked image

2. Determine the size of watermarked image

3. Use LSB of watermarked image to recover the watermark

4. Scale and display recovered watermark

\subsection{Algorithm 2}

The proposed algorithm in [6] presented a new LSB digital watermarking scheme by using random mapping function. The traditional LSB is usually embeds in the watermark bits by using a sequence order. However, an attacker can easily extract the watermark bits from the watermarked image. To remedy this problem, the proposed scheme adopts the randomly embedding technique using a random mapping function with seed. The proposed watermark schema is declared as being more robust than the traditional LSB technique.

\section{Embedding Algorithm}

1. Select the cover image (CI) and watermark image (WI).

2. Compute pixel number of the watermark image (WC).

3. Make pseudo random number as number of WC by using random mapping function with seed $\mathrm{k}$.

4. Generate coordinate of cover image by using random mapping function.

5. Embed watermark image to CI by performing the LSB technique.

\section{Extraction Algorithm}

1. Collect watermarked image (WI) and get seed $\mathrm{k}$.

2. Make pseudo random number as number of WC by using random mapping function with seed $\mathrm{k}$.

3. Generate coordinate of watermarked image by using random mapping function with seed $\mathrm{k}$.

4. Extract watermark image by performing the LSB technique.

\subsection{Algorithm 3}

The proposed watermarking schema in [7] investigates a tradeoff between imperceptibility and robustness of LSB watermarking by using the structural similarity index measure 
(SSIM) quality metrics. Significant bit planes of the watermark image are used instead of the lower bit-planes of image.

\section{Embedding Algorithm}

1. Read cover and watermark image.

2. Decomposed the two images into their 8 bit-planes.

3. Put $\mathrm{N}$ higher bit-planes of watermark image into $\mathrm{N}$ lower bit- planes of cover image.

4. This replacement has resulted in the watermarked image.

\section{Extraction Algorithm}

1. By using LSB extracted algorithm, extract the watermark image.

\subsection{Algorithm 4}

The proposed watermarking algorithm in [8], tries to embed the watermark data into the third and fourth LSBs. In this algorithm the grayscale image is read and transferred it into binary value and then it is embedded into the cover image to get the watermarked image.

\section{Embedding Algorithm}

1. Read the cover image and watermark data.

2. Convert the watermark data to binary values then specify the coordinates of the image where the data will be embedded.

3. Starting from the first coordinate embed the data into the third and fourth LSB.

4. Get the watermarked image.

\section{Extraction Algorithm}

1. Get the watermarked image.

2. Get the watermark data from the third and fourth LSB.

3. Convert the binary values of watermark data to characte to get the watermark data.

\subsection{Algorithm 5}

The proposed algorithm in [9] tries to achieve higher security and efficiency by combining two approaches: the digital watermarking and Cryptography for embedding the secret information. This algorithm enhances the security of the secret information hidden into the cover image. It implements an asymmetric key cryptography technique (RSA) and spatial domain technique in digital watermarking.

\section{Embedding Algorithm}

1. Choose two large prime numbers $p$ and $q$.
2. Calculates the private key $(\mathrm{d}, \mathrm{n})$ and public key by RSA (Rivest, Shamir and Adleman) algorithm.

3. By using the public key, encrypt the original image to produce Encrypted watermark image.

4. Embed the Encrypted watermark in the spatial domain of cover image and as a result Stego image is formed.

\section{Extraction Algorithm}

1. The encrypted watermark is extracted from the Stego image (Spatial domain)

2. By using the private key, decrypt the Encrypted watermarl and as a result original watermark is recovered.

\subsection{Algorithm 6}

The proposed watermarking schema in [12] is inversing the watermark bits before embedding it in original image. The proposed algorithm reads the cover image in grayscale image, and reads the watermark image, converts it into binary values and inverses its bits before embedding.

\section{Embedding Algorithm}

1- Read the watermark characters and convert it into bits.

2- Inverse the watermark bits.

3- Embed the watermark bits in the first LSB.

4- Save the watermarked image as bitmap image.

\section{Extraction Algorithm}

1- Read watermarked image.

2- Get the bits from the first LSB.

3- Inverse the bits and save into in array.

4- Convert the array to characters.

Table 1 summarizes the characteristics of the different LSB proposed algorithms depending on the papers themselves. 


\begin{tabular}{|c|c|c|c|c|c|c|}
\hline Parameter. & Algorithm1 & Algorithm2 & Algorithm3 & Algorithm4 & Algorithm5 & Algorithm6 \\
\hline $\begin{array}{c}\text { Main } \\
\text { concept }\end{array}$ & $\begin{array}{l}\text { Traditional LSB, } \\
\text { embed in LSB-1 } \\
\text { bits. }\end{array}$ & $\begin{array}{l}\text { Using randon } \\
\text { mapping } \\
\text { function. }\end{array}$ & $\begin{array}{l}\text { Using higher bit- } \\
\text { planes of the } \\
\text { watermark image } \\
\text { instead of lower } \\
\text { bit-planes. }\end{array}$ & $\begin{array}{l}\text { Using the third } \\
\text { and the fourth } \\
\text { least significan } \\
\text { bits (LSB) } \\
\text { technique. }\end{array}$ & $\begin{array}{c}\text { Encrypt watermark } \\
\text { by symmetric key } \\
\text { cryptography } \\
\text { technique (RSA). }\end{array}$ & $\begin{array}{c}\text { Watermark bits } \\
\text { inversed before } \\
\text { embedding into } \\
\text { image. }\end{array}$ \\
\hline Robustnes & Robust & $\begin{array}{c}\text { More } \\
\text { robustness }\end{array}$ & $\begin{array}{l}\text { Very low } \\
\text { robustness }\end{array}$ & More robustnes & Robust & Robust \\
\hline Security & $\begin{array}{l}\text { Watermark bits } \\
\text { easily extract by } \\
\text { attacker because } \\
\text { it's embedded in } \\
\text { sequence order. }\end{array}$ & More secure & $\begin{array}{l}\text { Watermark bits } \\
\text { easily extract by } \\
\text { attacker }\end{array}$ & $\begin{array}{l}\text { More secure, } \\
\text { hard to expect } \\
\text { that hidden dati } \\
\text { in 3rd\&4th LSE } \\
\text { bits }\end{array}$ & $\begin{array}{l}\text { Higher secrecy and } \\
\text { efficiency }\end{array}$ & $\begin{array}{c}\text { More secure, } \\
\text { because } \\
\text { watermark bits is } \\
\text { inversed. }\end{array}$ \\
\hline Fidelity & Higher & Higher & Lower & Higher & Higher & Higher \\
\hline
\end{tabular}

\section{COMPARISON TOOLS}

This section introduces some tools used to compare the results of the different LSB proposed algorithms. To be able to do so, proposed algorithms implemented correctly and same input images used. We used four images as a standard evaluation for watermarking algorithms. These Images are shown in figure 1 . All images are grey scale image in different sizes. The watermark image used for embedding is shown in figure 2 . There are a numerous measures used to determine the effectiveness of watermarking algorithm and which directly or indirectly influences the properties of inserted watermark. To calculate the quality performance of watermarked image, five of the measurement tools were used, which are:

- Mean Square Error (MSE): represents the cumulative squared error between the watermarked image and the original image. In ideal case MSE should be zero. The smaller value of the MSE represents the better result. It is calculated in equation 1 . Where $\mathrm{A}$ is the original image, $\mathrm{B}$ is watermarked image, $\mathrm{m} \times \mathrm{n}$ is the size of both images.

$$
M S E=\frac{1}{m n} \sum_{i=1}^{m} \sum_{j=1}^{n}(A i j-B i j)^{2}
$$

- Peak signal-to-noise ratio (PSNR): represents a measure of the peak error. We use PSNR to measure the visual fidelity or imperceptibility between the original image and the watermarked image. The greater value of the PSNR means better quality of watermarked image. It is given in equation 2. Where Max is the maximum value in host image.

$$
\text { PSNR }=10 \log \left(\frac{\mathrm{Max}^{2}}{\mathrm{MSE}}\right)
$$

- Normalized cross correlation (NCC): is utilized to check the robustness of the proposed watermarking schemas against different attacks. The NCC is utilized to show the likeness between two images. For our situation, it is used to decide how much the extracted watermark is close to the original watermark image. On the off chance that the NCC between the two images is 1 , that implies both images are the same. It is calculated using the equation 3. Where, Aij represents the original image pixels and $\mathrm{Bij}$ represents watermarked image pixels.

$$
N C C==\frac{\sum_{i=1}^{m} \sum_{j=1}^{n} A i j B i j}{\sum_{i=1}^{m} \sum_{j=1}^{n}(A i j)^{2}}
$$

- Average difference (AD): is simply the average of difference between the original image and

watermarked image. It is calculated by the equation 4 . Where $\mathrm{x}$ is the original image, $\mathrm{x}$ ' is watermarked image and $\mathrm{MxN}$ is the size of original image.

$$
\mathbf{A D}=\sum_{\mathbf{j}=\mathbf{1}}^{\mathrm{M}} \sum_{\mathrm{k}=\mathbf{1}}^{\mathrm{N}} \frac{\left(\mathbf{X j}, \mathbf{k}-\mathbf{X}^{\prime} \mathbf{j}, \mathbf{k}\right)}{\mathrm{MN}}
$$

- Normalized absolute error (NAE): It should be the minimum in order to minimize the difference between original and watermarked image. A higher NAE value shows that the image is of poor quality. It is calculated by the equation 5 . Where $\mathrm{x}$ is the original image, and $\mathrm{x}$ ' is watermarked image.

$$
\mathrm{NAE}=\sum_{\mathrm{j}=1}^{\mathrm{M}} \sum_{\mathrm{k}=1}^{\mathrm{N}} \frac{\left|\mathrm{Xj}, \mathrm{k}-\mathrm{X}^{\prime} \mathrm{j}, \mathrm{k}\right|}{\sum_{\mathrm{j}=1}^{\mathrm{M}} \sum_{\mathrm{k}=1}^{\mathrm{N}}|\mathrm{Xj}, \mathrm{k}|}
$$

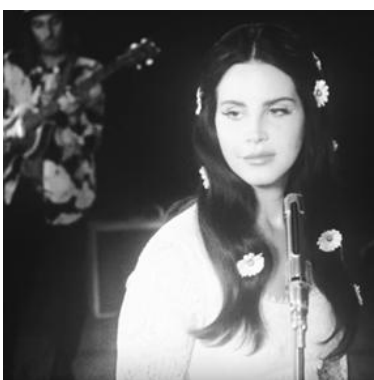

image 1

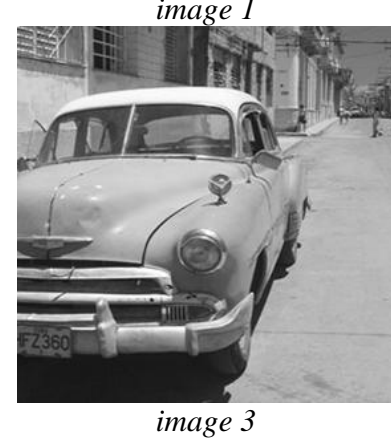

Figure 1: the four original images

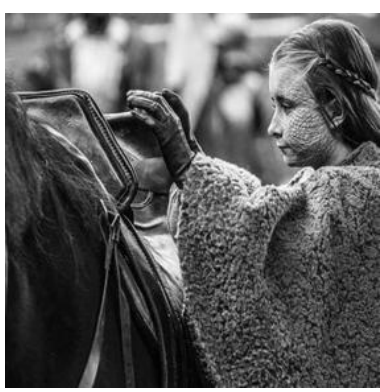

image 2

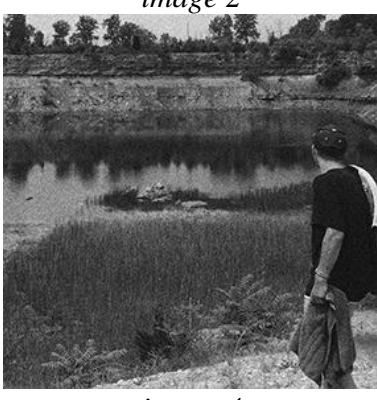

image 4 


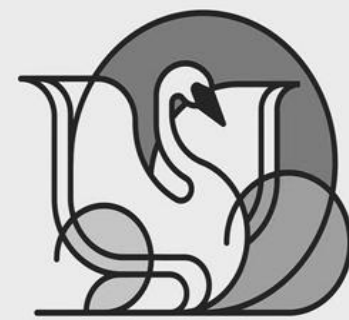

Figure 2: watermark image

\section{EXPERIMENTAL RESULTS}

After implementing the LSB's algorithms we noticed that, for all algorithms and images, there is no visual difference between the original and watermarked images. No distortion occurs for watermarked images. This is mainly because the modifications occur on the LSBs.

According to the results shown in table 2, Algorithm 4 has an MSE very high. There is an inverse relationship between PSNR and MSE. So higher MSE value indicates the lower quality of the image. Typical values for the PSNR are between $30 \mathrm{~dB}$ and $40 \mathrm{~dB}$. If the PSNR value of the watermarked image is greater than 30 , it is difficult to notice the difference between the cover and watermarked images. Algorithm 1 has higher value of PSNR means good image quality and less error introduced to the image. The AD value and NAE value are higher for Algorithm 4 that indicate that watermarked image is of poor quality. The NCC values of algorithms are almost 1 that is normal as the original and extracted watermarks should be similar in the absence of attacks. Algorithm 1 and 6 show the better results among all these algorithms.

Table 2: Performance for the different algorithms

\begin{tabular}{|c|c|c|c|c|c|}
\hline \multicolumn{6}{|c|}{ Algorithm1 } \\
\hline Image & MSE & PSNR & $\mathrm{NCC}$ & $\mathrm{AD}$ & NAE \\
\hline Image 1 & 0.5097 & 55.8288 & 0.9669 & 0.2976 & 0.0018 \\
\hline Image2 & 0.5006 & 55.9070 & 0.9669 & 0.2841 & 0.0019 \\
\hline Image 3 & 0.5031 & 55.8856 & 0.9669 & 0.2826 & 0.0012 \\
\hline Image4 & 0.5001 & 55.9113 & 0.9669 & 0.2834 & 0.0020 \\
\hline \multicolumn{6}{|c|}{ Algorithm2 } \\
\hline Image & MSE & PSNR & $\mathrm{NCC}$ & $\mathrm{AD}$ & NAE \\
\hline Image 1 & 0.5084 & 51.0687 & 1.0000 & 0.3076 & 0.0054 \\
\hline Image2 & 0.4999 & 51.1419 & 1.0000 & 0.2942 & 0.0057 \\
\hline Image 3 & 0.5023 & 51.1208 & 1.0000 & 0.2927 & 0.0037 \\
\hline Image4 & 0.5005 & 51.1367 & 1.0000 & 0.2934 & 0.0060 \\
\hline \multicolumn{6}{|c|}{ Algorithm3 } \\
\hline Image & MSE & PSNR & $\mathrm{NCC}$ & $\mathrm{AD}$ & NAE \\
\hline Image 1 & 0.5093 & 51.0609 & 1.0000 & 0.3076 & 0.0054 \\
\hline
\end{tabular}

\begin{tabular}{|c|c|c|c|c|}
\hline Image 2 & 0.5000 & $51.1412 \quad 1.0000$ & 0.2942 & 0.0057 \\
\hline Image3 & 0.5018 & $51.1253 \quad 1.0000$ & 0.2927 & 0.0037 \\
\hline Image 4 & 0.5019 & $51.1249 \quad 1.0000$ & 0.2934 & 0.0061 \\
\hline \multicolumn{5}{|c|}{ Algorithm4 } \\
\hline Image & MSE & PSNR NCC & $\mathrm{AD}$ & NAE \\
\hline Image 1 & 5.1438 & $41.0179 \quad 1.0000$ & 1.0416 & 0.0111 \\
\hline Image2 & 5.4625 & $40.7569 \quad 1.0000$ & 1.1058 & 0.0128 \\
\hline Image 3 & 5.3917 & $40.8136 \quad 1.0000$ & 1.0928 & 0.0081 \\
\hline Image4 & 5.4444 & $40.7713 \quad 1.0000$ & 1.0994 & 0.0134 \\
\hline \multicolumn{5}{|c|}{ Algorithm5 } \\
\hline Image & MSE & PSNR NCC & $\mathrm{AD}$ & NAE \\
\hline Image 1 & 0.5083 & $\begin{array}{ll}51.0695 & 1.0000\end{array}$ & 0.3076 & 0.0054 \\
\hline Image2 & 0.4997 & $51.1436 \quad 1.0000$ & 0.2942 & 0.0057 \\
\hline Image 3 & 0.4988 & $51.1516 \quad 1.0000$ & 0.2927 & 0.0037 \\
\hline Image 4 & 0.5011 & $51.1315 \quad 1.0000$ & 0.2934 & 0.0061 \\
\hline \multicolumn{5}{|c|}{ Algorithm6 } \\
\hline Image & MSE & PSNR NCC & $\mathrm{AD}$ & NAE \\
\hline Image 1 & 0.2526 & 54.10701 .0000 & 0.2526 & 0.0027 \\
\hline Image2 & 0.2495 & $\begin{array}{ll}54.1598 & 0.9999\end{array}$ & 0.2495 & 0.0029 \\
\hline Image 3 & 0.2515 & $54.1252 \quad 1.0000$ & 0.2515 & 0.0019 \\
\hline Image4 & 0.2497 & 54.15590 .9998 & 0.2497 & 0.0030 \\
\hline
\end{tabular}

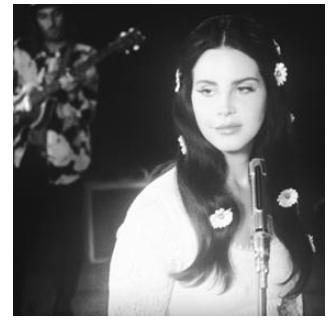

1 Image

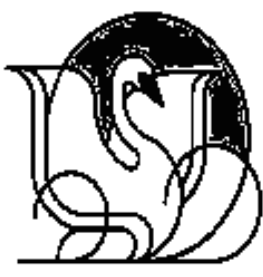

watermark

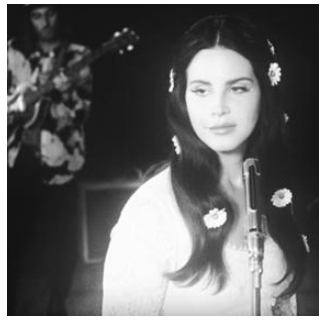

watermarked Image

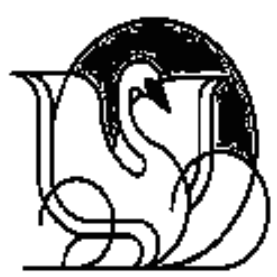

Extracted watermark
Figure 3: Algorithm 1 results 


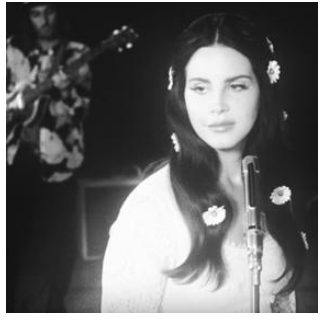

1 Image

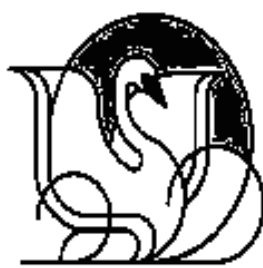

watermark

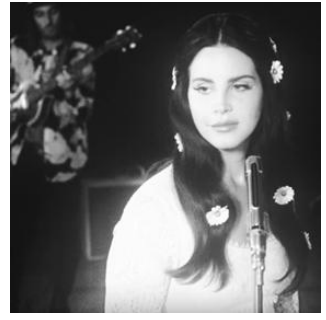

watermarked Image

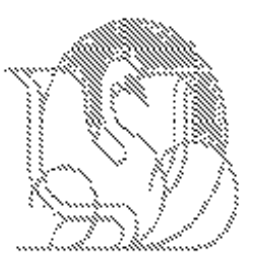

Extracted watermark
Figure 4: Algorithm 4 results

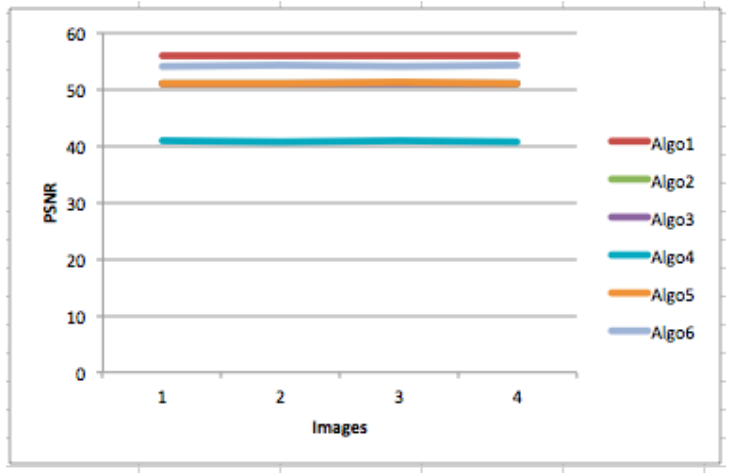

Figure 5: PSNR values analysis

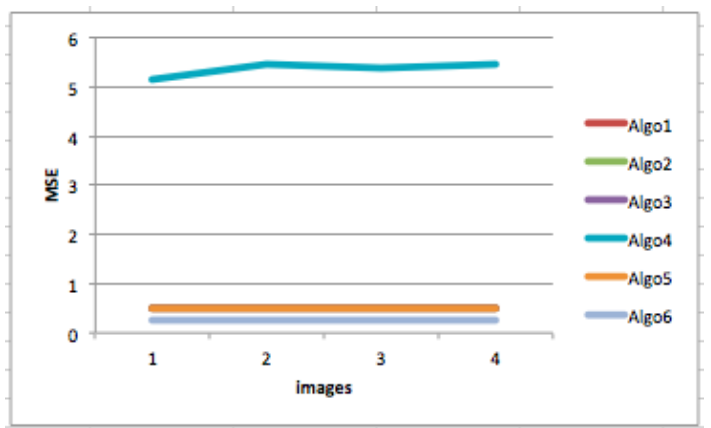

Figure 6: MSE values analysis

Figures 5 and 6 show clearly that Algorithm 4 is the worst algorithm and all the rest have good PSNR.

\section{CROPPING ATTACK}

One of the attacks in spatial domain is cropping attack. It occurs when parts of the watermarked image is cropped by the attacker which effect on watermarked information. Figure 7 shows the watermarked image after a cropping attack. The NCC calculated for the extracted watermark from cropped watermarked image for each algorithm are shown in table 3, table 4 and table 5 .
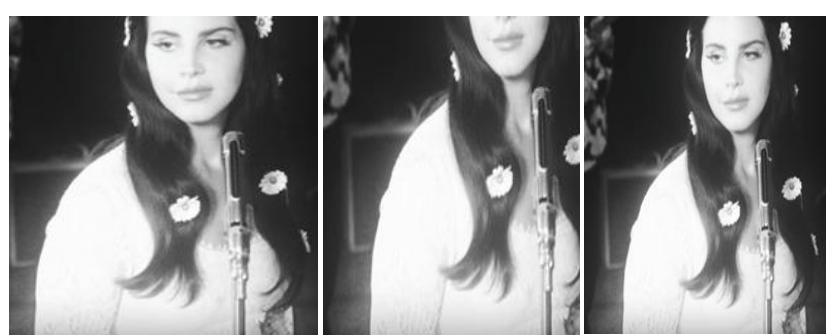
a) corpping
size[70,60,300,400]

b) corpping size $[50,100,205,41$ s

c) corpping size ge

Table 3: NCC results after cropping attack (a)

\begin{tabular}{lllll}
\hline & Image 1 & Image 2 & Image 3 & Image 4 \\
\hline Algorithm 1 & 0.9353 & 0.9004 & 0.9353 & 0.9004 \\
\hline Algorithm 2 & 0.4966 & 0.4980 & 0.5980 & 0.5884 \\
\hline Algorithm 3 & 0.2250 & 0.3069 & 0.3335 & 0.3098 \\
\hline Algorithm 4 & 0.3369 & 0.3362 & 0.3371 & 0.3353 \\
\hline Algorithm 5 & 0.6420 & 0.9815 & 0.7142 & 0.9094 \\
\hline Algorithm 6 & 0.7469 & 0.8245 & 0.8005 & 0.9171 \\
\hline
\end{tabular}

Table 4: NCC results after cropping attack (b)

\begin{tabular}{llccc}
\hline & Image 1 & Image 2 & Image 3 & Image 4 \\
\hline Algorithm 1 & 0.9461 & 0.9240 & 0.9461 & 0.9240 \\
\hline Algorithm 2 & 0.4966 & 0.5801 & 0.5775 & 0.5792 \\
\hline Algorithm 3 & 0.3315 & 0.3081 & 0.3348 & 0.3072 \\
\hline Algorithm 4 & 0.3376 & 0.3351 & 0.3363 & 0.3351 \\
\hline Algorithm 5 & 0.6404 & 0.9766 & 0.6980 & 0.8924 \\
\hline Algorithm 6 & 0.7469 & 0.9233 & 0.9341 & 0.8183 \\
\hline
\end{tabular}

Table 5: NCC results after cropping attack (c)

\begin{tabular}{llccc}
\hline & Image 1 & Image 2 & Image 3 & Image 4 \\
\hline Algorithm 1 & 0.9248 & 0.9248 & 0.9248 & 0.9248 \\
& & & & \\
\hline Algorithm 2 & 0.4966 & 0.5915 & 0.5992 & 0.5881 \\
\hline Algorithm 3 & 0.3082 & 0.3081 & 0.3029 & 0.3095 \\
\hline Algorithm 4 & 0.3339 & 0.3352 & 0.3382 & 0.3359 \\
\hline Algorithm 5 & 0.6914 & 0.9805 & 0.7688 & 0.8942 \\
\hline Algorithm 6 & 0.7398 & 0.8288 & 0.8288 & 0.9185
\end{tabular}

As can be clearly seen in the three tables 3,4 , and 5 the Algorithms 1 and 6 maintain their robustness and the quality of all the other algorithms is dropping down. As the cropping is mainly done in watermarked image sides, this result indicates 
that the Algorithms 1 and 6 do not use a lot the sides' pixels to embed the information.

\section{CONCLUSION}

This paper studies different proposed LSB watermarking algorithms and compares their performances. The well-known weakness in LBS watermark algorithms is their weak robustness against attacks. Most of the papers claim that their proposed algorithms are robust against attacks while they are not providing performance results after introducing some attacks. Our comparison is then done without introducing the attacks that impact the least significant bits that destroy the watermark information. The comparison was mainly taking into account the watermarked image quality. Algorithm 1 seems to be the best providing such good quality while algorithm 4 was the worst.

After performing cropping attack, its clearly that watermark is destroyed considerably. The Algorithms 1 and 6 are the only ones that maintain their robustness against this attack as shown in the results provided in table 3 , table 4 and table 5 . The clustering used in [16-18] to duplicate the embedded information over dispersed pixels may be a solution to avoid such robustness deterioration when getting such attacks.

The future work will concentrate on the frequency domain techniques. These techniques are known by their robustness, as the information is not embedded into the LSB. Their complexity and low capacity are declared as the main drawbacks.

\section{ACKNOWLEDGEMENT}

This work was supported in part by the Deanship of Scientific Research, Qassiem University, under Grant 2029-coc-2016-1-12S. Authors gratefully acknowledged this support.

\section{REFERENCES}

[1] Kurak, Charles, and John McHugh. "A cautionary note on image downgrading." Computer Security Applications Conference, 1992. Proceedings. Eighth Annual. IEEE, 1992.

[2] Zheng, Dong, et al. "A survey of RST invariant image watermarking algorithms." ACM Computing Surveys (CSUR) 39.2 (2007): 5 .

[3] Yang, Wen-Chao, Che-Yen Wen, and Chung-Hao Chen. "Applying public-key watermarking techniques in forensic imaging to preserve the authenticity of the evidence." International Conference on Intelligence and Security Informatics. Springer, Berlin, Heidelberg, 2008.

[4] He, HongJie, JiaShu Zhang, and Heng-Ming Tai. "A wavelet-based fragile watermarking scheme for secure image authentication." International Workshop on Digital Watermarking. Springer, Berlin, Heidelberg, 2006.

[5] Byun, Sung-Cheal, et al. "A SVD-based fragile watermarking scheme for image authentication." International Workshop on Digital Watermarking. Springer, Berlin, Heidelberg, 2002.

[6] Lee, Gil-Je, Eun-Jun Yoon, and Kee-Young Yoo. "A new LSB based digital watermarking scheme with random mapping function." Ubiquitous Multimedia Computing, 2008. UMC'08. International Symposium on. IEEE, 2008.

[7] Fazli, Saeid, and Gholamreza Khodaverdi. "Trade-off between imperceptibility and robustness of LSB watermarking using SSIM quality metrics." Machine Vision, 2009. ICMV'09. Second International Conference on. IEEE, 2009.
[8] Bamatraf Abdullah, Rosziati Ibrahim, and Mohd Najib B. Mohd Salleh. "Digital watermarking algorithm using LSB." Computer Applications and Industrial Electronics (ICCAIE), 2010 International Conference on. IEEE, 2010.

[9] Singh Krishna Kumar, and Shashank Dwivedi. "Digital Watermarking using Asymmetric Key Cryptography and Spatial Domain Technique." International Journal of Advance Research in Computer Science and Management Studies 2.8 (2014): 65-72.

[10] Low, Cheng-Yaw, Andrew Beng-Jin Teoh, and Connie Tee. "Fusion of LSB and DWT biometric watermarking using offline handwritten signature for copyright protection." International Conference on Biometrics. Springer, Berlin, Heidelberg, 2009.

[11] El Kerek Bilal, et al. "A new technique to multiplex stereo images: LSB watermarking and Hamming code." Artificial Intelligence, Modelling and Simulation (AIMS), 2013 1st International Conference on. IEEE, 2013.

[12] Bamatraf, Abdullah, et al. "A new digital watermarking algorithm using combination of least significant bit (LSB) and inverse bit." arXiv preprint arXiv:1111.6727 (2011).

[13] Kumar Sanjeev, and Tanupreet Singh. "Performance improvement of simple LSB watermarking using SVD." Computational Intelligence on Power, Energy and Controls with their impact on Humanity (CIPECH), 2014 Innovative Applications of. IEEE, 2014.

[14] Dehkordi, Amin Banitalebi, Said Nader Esfahani, and Alireza Nasiri Avanaki. "Robust LSB watermarking optimized for local structural similarity." Electrical Engineering (ICEE), 2011 19th Iranian Conference on. IEEE, 2011

[15] Apostol, Cristian-Gabriel, and Cristian-Iulian Rîncu. "Digital watermarking secured with PWLCM, chaoticfeedback and LSB data hiding." Communications (COMM), 2010 8th International Conference on. IEEE, 2010.

[16] Mohamed Tahar Ben Othman, "Novel image clustering based on image features for robust reversible data hiding", INTERNATIONAL JOURNAL of FUZZY SYSTEMS and ADVANCED APPLICATIONS 2, 2015, pp. 1-8.

[17] Mohamed Tahar Ben Othman, "Digital Image Watermarking based on image clustering", the 3rd International Conference on Circuits, Systems, Communications, Computers and Applications (CSCCA '14),. Florence, Italy, November 22-24, 2014.

[18] Mohamed Tahar Ben Othman, "CAM-based Digital Image Watermarking Revisited", WSEAS Transaction on Systems, 2014/1.

[19] Mohamed Tahar Ben Othman, New Image Watermarking Scheme based on Image Content Addressing Method", The 13th WSEAS International Conference on Applied Computer and Applied Computational Science, 23-25 April 2014 